\title{
Identification and properties of the Deinococcus grandis and Deinococcus proteolyticus single-stranded DNA binding proteins (SSB)
}

\author{
Paweł Filipkowski and Józef Kur ${ }^{\bigotimes}$ \\ Gdańsk University of Technology, Chemical Faculty, Department of Microbiology, Gdańsk, Poland \\ Received: 18 September, 2006; revised: 03 January, 2007; accepted: 18 January, 2007 \\ available on-line: 27 February, 2007
}

\begin{abstract}
To study the biochemical properties of SSB's from Deinococcus grandis (DgrSSB) and Deinococcus proteolyticus (DprSSB), we have cloned the ssb genes obtained by PCR and have developed Escherichia coli overexpression systems. The genes consist of an open reading frame of 891 ( $D g r S S B)$ and 876 (DprSSB) nucleotides encoding proteins of 296 and 291 amino acids with a calculated molecular mass of 32.29 and $31.33 \mathrm{kDa}$, respectively. The amino-acid sequence of $\mathrm{DgrSSB}$ exhibits $45 \%, 44 \%$ and $82 \%$ identity and the amino-acid sequence of $D p r S S B$ reveals $43 \%, 43 \%$ and $69 \%$ identity with Thermus aquaticus (TaqSSB), Thermus thermophilus (TthSSB) and Deinococcus radiodurans SSBs, respectively. We show that DgrSSB and DprSSB are similar to Thermus/ Deinococcus SSBs in their biochemical properties. They are functional as homodimers, with each monomer encoding two single-stranded DNA binding domains (OB-folds). In fluorescence titrations with poly(dT), both proteins bind single-stranded DNA with a binding site size of about $33 \mathrm{nt}$ per homodimer. In a complementation assay in E. coli, DgrSSB and DprSSB took over the in vivo function of EcoSSB. Thermostability with half-lives of about $1 \mathrm{~min}$ at $65-67.5^{\circ} \mathrm{C}$ make DgrSSB and DprSSB similar to the known SSB of Deinococcus radiodurans (DraSSB).
\end{abstract}

Keywords: SSB, DNA replication, expression, purification, bacteria

\section{INTRODUCTION}

Single-stranded DNA-binding (SSB) proteins play central roles in cellular and viral processes involving the generation of single-stranded DNA. These include DNA replication, DNA repair pathways and homologous recombination (Shamoo et al., 1995; Webster et al., 1997; Wadsworth \& White, 2001; Kur et al., 2005). SSBs bind DNA using four 'OB-fold' (oligonucleotide/oligosaccharide-binding fold) domains (Murzin, 1993) that can be organized in a variety of overall quaternary structures. They are found in different organisms as homotetramers (most prokaryotic, mitochondrial and crenarchaeal SSBs), homodimers (SSBs from bacteriophages, Deinococcus-Thermus phylum of bacteria), heterotrimers (euryarchaeal and eucaryal RPAs)
(Williams \& Konigsberg, 1978; Shamoo et al., 1995; Stassen et al., 1995; Webster et al., 1997; Wadsworth \& White, 2001; Dąbrowski et al., 2002a; Bernstein et al., 2004; Kur et al., 2005). Although there is little conservation at sequence level, the crystal structures of human RPA (Bochkarev et al., 1997; 1999), Escherichia coli (Webster et al., 1997; Raghunathan et al., 2000), Deinococcus radiodurans (Bernstein et al., 2004), Thermus aquaticus (Jedrzejczak et al., 2006) and human mitochondrial (Yang et al., 1997) SSBs indicate the presence of a core 'OBfold'. This domain comprises approximately 100 amino acids and is responsible for the major interactions with single-stranded DNA. The universality of OB-fold domains in SSB proteins suggests that all extant SSB and RPA proteins are derived from a common ancestral SSB.

\footnotetext{
${ }^{\square}$ Author for correspondence: Józef Kur, Gdańsk University of Technology, Chemical Faculty, Department of Microbiology, Gdańsk, Poland; tel.: (48 58) 347 2302; fax: (48 58) 347 2694; e-mail: kur@chem.pg.gda.pl

Nucleotide sequence accession number. The nucleotide sequences of the ssb genes are available in the Gen Bank database under accession numbers: for D. grandis: DQ857725 and for D. proteolyticus: DQ979351.

Abbreviations: dsDNA, double-stranded DNA; IPTG, isopropyl $\beta$-D-thiogalactoside; OB fold, oligonucleotide/oligosaccharide-binding fold; RPA, replication protein A; SSB, single-stranded DNA binding protein.
} 
Although, virtually all bacterial SSB family members act as homotetramers, recent discoveries have shown that SSB proteins from Deinococcus-Thermus phylum of bacteria adopt a different architecture. They are functional as homodimers, with each SSB monomer encoding two OB folds linked by a conserved spacer sequence (Dąbrowski et al., 2002a; Bernstein et al., 2004; Kur et al., 2005). Homodimeric SSBs, contrary to tetrameric SSB proteins, possess only two C-terminal tails in each active form. Reducing the number of C-terminal tails by half can dramatically affect the function of SSB protein containing two OB-folds in vivo (Curth et al., 1996). Probably the fusion of two OB folds linked by a conserved spacer sequence is an adaptation of Deinococcus-Thermus to extreme conditions (Dąbrowski et al., 2002a; 2002b; Bernstein et al., 2004). Bacteria from this group thrive in extreme environments that would kill most cells (desiccation, severe DNA-damaging and/or high-temperature conditions) by using mechanisms that are presently unclear.

At the present time there are twenty validly described Deinococcus species (Hirsch et al., 2004; de Groot et al., 2005; Omelchenko et al., 2005). With the exception of $D$. murrayi and D. geothermalis, which have optimum growth temperatures of 45 to $50^{\circ} \mathrm{C}$, all other species have optimum growth temperatures between about 25 to $35^{\circ} \mathrm{C}$ (Ferreira et al., 1997). The most striking characteristic of the species of this genus is their extreme resistance to UV and gamma radiation. Two Deinococcus genome sequences are currently available ( $D$. radiodurans and $D$. geothermalis) and SSBs of D. radiodurans, D. radiopugnans and D. geothermalis have been described in detail (Bernstein et al., 2004; Witte et al., 2005).

At present three Deinococcus SSB-like proteins have been described in detail. They are SSBs from D. radiodurans (Bernstein et al., 2004; Witte et al., 2005), D. geothermalis (Filipkowski et al., 2006a) and D. radiopugnans (Filipkowski et al., 2006b). Here we report the identification, purification and characterization of new SSB proteins from other Deinococcus species (D. grandis and D. proteolyticus), and their relationship with other members of this important class of proteins.

\section{MATERIALS AND METHODS}

Bacterial strains, plasmids, enzymes, and reagents. D. grandis DSMZ 3963 and D. proteolyticus DSMZ 20540 strains were purchased from DSMZ (Deutsche Sammlung von Mikroorganismen und Zellkulturen $\mathrm{GmbH}$, Germany). E. coli TOP10F' strain and the pBADMycHisA plasmid were from Invitrogen (USA) and were used for protein expression. The reagents for PCR and various oligodeoxy- nucleotides were obtained from DNA-Gdańsk II (Poland). DNA from D. grandis and D. proteolyticus were isolated using Genomic DNA Prep Kit (A\&A Biotechnology, Poland).

Identification of $D$. grandis and D. proteolyticus ssb genes. Based on the known localization of the ssb gene in D. radiodurans (GenBank Accession No. AY293617) and D. geothermalis 11300 (GenBank Accession No. NC008025 and CP000359, previously: AAHE01000002) genomes, where the ssb gene is flanked by the conservative $r p s F$ and $r p s R$ genes encoding the ribosomal proteins S6 and S18, primers complementary to those genes for PCR amplification were designed and synthesized. The forward primer used for amplification of DNA containing the ssb gene of $D$. grandis was: 5'-GACAACGTCCGCCGCGTCCTGGTGGT (26 nt) and the reverse primer was: 5'-GTGCGGCGGCGGGGAAGAATCTT (23 nt) (Filipkowski et al., 2006a). The forward primer used for PCR amplification of DNA containing the ssb gene of D. proteolyticus was: 5'-GCCCRGARTGGAAGACCAAGAAGGC (25 nt) and the reverse primer was: 5'-GCGGCGGGGAAGAATCTTGCC (21 nt) (Filipkowski et al., 2006b). PCRs were carried out using proofreading DNA polymerase Pwo (DNA-Gdańsk II, Poland) and 35 cycles with a temperature profile of $60 \mathrm{~s}$ at $95^{\circ} \mathrm{C}, 60 \mathrm{~s}$ at $68^{\circ} \mathrm{C}$, and $90 \mathrm{~s}$ at $72^{\circ} \mathrm{C}$. In both cases specifically, about 1200 $1300 \mathrm{bp}$ PCR products were obtained and then were sequenced to confirm the presence of ssb-like gene.

The obtained ssb gene sequences of $D$. grandis and $D$. proteolyticus were used to design gene-specific primers for PCR and cloning into pBADMycHisA vector. PCR was carried out using forward 5'-ATACCATGGCCCGAGGCATGAACCA- $3^{\prime}$ and reverse 5'-A AAGCTTCAGAACGGCAGGTCCTCGTC-3' primers for $s s b$ of $D$. grandis and forward 5'-TATACCATGGCCCGAGGAATGAATCACGTT- ${ }^{\prime}$ and reverse 5'-ACTAAGCTTCAAAACGGCATATCGTCTTCTTCTGG-3' primers for ssb of D. proteolyticus. The boldface parts of primer sequences are complementary to the nucleotide sequences of the ssb gene, whereas $5^{\prime}$ overhanging ends of primers contain recognition sites for restriction endonucleases and are designed to facilitate cloning ( $\mathrm{NcoI}$ or HindIII sites, underlined; start and stop codons, italicized). For $D$. grandis ssb amplification 25 cycles were performed with a temperature profile of $60 \mathrm{~s}$ at $94^{\circ} \mathrm{C}, 60 \mathrm{~s}$ at $68.4^{\circ} \mathrm{C}$, and $120 \mathrm{~s}$ at $72^{\circ} \mathrm{C}$ and for D. proteolyticus ssb amplification 30 cycles were performed with a temperature profile of $60 \mathrm{~s}$ at $94^{\circ} \mathrm{C}, 60 \mathrm{~s}$ at $67.7^{\circ} \mathrm{C}$, and $120 \mathrm{~s}$ at $72^{\circ} \mathrm{C}$.

Cloning of D. grandis and D. proteolyticus $s s b$ genes. The obtained PCR products were digested with NcoI and HindIII, and fractionated on 1.5\% agarose gel. DNA corresponding to $902 \mathrm{bp}$ for $D$. grandis ssb and $890 \mathrm{bp}$ for $D$. proteolyticus ssb were 
cut out of the gel and purified using Gel-Out Kit (A\&A Biotechnology, Poland). In both cases the purified fragments were ligated into pBADMycHisA between NcoI and HindIII sites. E. coli TOP10F' cells were transformed with the ligation mixture and obtained colonies were examined for the presence of $s s b$ gene of $D$. grandis or D. proteolyticus by PCR amplification and restriction analysis. The resulting plasmid was referred to as pBADDgrSSB or pBADDprSSB. The authenticity of the clones was ascertained by sequencing.

Expression and purification. SSBs were overexpressed using pBADDgrSSB or pBADDprSSB in E. coli TOP10F' as described previously (Filipkowski et al., 2006a) with some modifications. Cells were grown in $500 \mathrm{ml}$ culture at $37^{\circ} \mathrm{C}$ to an $\mathrm{OD}_{600}$ of 0.6 in LB-broth containing $100 \mu \mathrm{g} / \mathrm{ml}$ ampicillin. The expression of SSBs was induced with arabinose at a final concentration of $0.2 \%$ for $\mathrm{DgrSSB}$ and $0.02 \%$ for DprSSB. Cells were harvested after additional $4 \mathrm{~h}$ culturing by centrifugation and suspended in $30 \mathrm{ml}$ buffer A consisting of $10 \mathrm{mM}$ Tris/ $\mathrm{HCl}, \mathrm{pH} 9,10$ $\mathrm{mM}$ EDTA, $10 \mathrm{mM} \mathrm{CaCl}{ }_{2}, 0.5 \mathrm{mg} / \mathrm{ml}$ lysozyme, and lysed by sonication.

The purification procedure was analogous to the previously published purification scheme for thermostable SSB-like proteins (Dąbrowski et al., 2002) with some modifications in the first step of procedure (heat treatment). The reduction of contamination by the host proteins after heat treatment was carried out at $57.5^{\circ} \mathrm{C}$ for $10 \mathrm{~min}$. The purity of proteins was examined by SDS/PAGE. The amounts of the purified SSB protein were estimated spectrophotometrically using the appropriate absorption coefficient factor and by the optical densitometry on SDS/PAGE gel with Quantity One program (VersaDoc, BioRad).

The cloning, expression and purification procedure for SSB from $D$. radiodurans (DraSSB) was analogous to the previously published scheme by Eggington et al. (2004).

Analytical gel filtration chromatography. DgrSSB and DprSSB proteins (up to about $1 \mathrm{mg}$ total) were subjected to gel filtration chromatography on a Superdex HR 75 column (Amersham Bioscence) equilibrated with $60 \mathrm{ml}$ buffer containing $20 \mathrm{mM}$ Tris/ $\mathrm{HCl}, \mathrm{pH}$ 7.5, $150 \mathrm{mM} \mathrm{NaCl}, 10 \mathrm{mM}$ EDTA, and were eluted with the same buffer at a flow rate of $0.5 \mathrm{ml} / \mathrm{min}$ and were collected in $2.5 \mathrm{ml}$ fractions. Absorbance at $280 \mathrm{~nm}$ was recorded to monitor the elution profile. The elution patterns of SSB proteins were then compared with those of standard proteins: bovine albumin $(66 \mathrm{kDa})$, ovalbumin $(43 \mathrm{kDa})$, carbonic anhydrase $(29 \mathrm{kDa})$ and cytochrome $c(12.4$ $\mathrm{kDa})$.

Agarose gel electrophoretic retardation analysis. The fluorescein-labelled ss dT-oligonucleotides
$35,60,70$ or 76 nucleotides in length $(10 \mathrm{pM})$ were incubated with a known concentration of purified SSB protein in binding buffer $(20 \mathrm{mM}$ Tris/ $\mathrm{HCl}$, $\mathrm{pH} 7.5,10 \mathrm{mM}$ EDTA) containing 2 or $100 \mathrm{mM} \mathrm{NaCl}$. After $20 \mathrm{~min}$ at $25^{\circ} \mathrm{C}$ samples were loaded onto $2 \%$ agarose gels and separated by electrophoresis in $1 \times$ TAE buffer. The complexes and the free oligonucleotides were visualized under UV light.

Fluorescence titrations. The fluorimetric titration experiments were performed on a Perkin-Elmer LS-5B luminescence spectrometer as described earlier (Dąbrowski \& Kur, 1999). The tryptophan emission spectra of the examined SSB proteins were obtained by excitation at $295 \mathrm{~nm}$ in reaction buffer $(20 \mathrm{mM}$ Tris/ $\mathrm{HCl}, \mathrm{pH} 7.5,10 \mathrm{mM}$ EDTA) containing 2 or $100 \mathrm{mM} \mathrm{NaCl}$ at $25^{\circ} \mathrm{C}$. Fluorescence quenching at the emission peak of $348 \mathrm{~nm}$ was monitored whilst titrating $(\mathrm{dT})_{60}$ oligonucleotide into a fixed concentration of SSB protein. Relative fluorescence values were corrected for dilution effects and subtraction of fluorescence due to buffer and DNA components.

Thermostability. A fixed quantity $(10 \mathrm{pM})$ of 5 '-end fluorescein-labelled oligonucleotide $(\mathrm{dT})_{35}$ was added to $10 \mathrm{pM}$ of DgrSSB,DprSSB, DraSSB or TaqSSB samples preincubated for $0,1,5,8,10,30$, $20,30,40,60$, and $90 \mathrm{~min}$ at $50,55,60,65$ and $67.5^{\circ} \mathrm{C}$ in $10 \mu \mathrm{l}$ reaction mixtures in standard buffer B. After $20 \mathrm{~min}$ incubation at $25^{\circ} \mathrm{C}$ protein-DNA complexes were separated from free DNA by $2 \%$ agarose gel electrophoresis, and $50 \%$ quantities of protein- $(\mathrm{dT})_{35}$ complex were evaluated by densitometric analysis using VersaDoc imaging system and QuantityOne software (BioRad).

Complementation analysis. E. coli RDP268 strain, in which the chromosomal ssb gene is replaced by a kanamycin resistance $\left(\Delta s s b:: \mathrm{Kan}^{\mathrm{R}}\right)$ and harboring pRPZ146 (ColEl ori, $\mathrm{Tc}^{\mathrm{R}}$ ) plasmid coding the wild-type SSB protein (Porter \& Black, 1991) was lysogenized with $\lambda$ DE3 using $\lambda$ DE3 Lysogenization Kit (Novagen). The resulting strain can be used to express target genes cloned in the pET vector under the control of the T7 promoter. pRPZ146 is essential for the survival of the cells and can be replaced by another plasmid, only if it contains a gene whose product can take over EcoSSB function in vivo. We used the modified E. coli RDP268(DE3) strain to transform the plasmids pET23D (+)DgrSSB, pET23D (+)DprSSB or pET23D $(+) E \operatorname{coSSB}$, which carries the ssb genes under the control of the T7 promoter and confers ampicillin resistance. Strains were grown in the presence of ampicillin $(100 \mu \mathrm{g} / \mathrm{ml})$ and kanamycin $(25 \mu \mathrm{g} / \mathrm{ml})$ - present during all steps. After four consecutive overnight subculturings in $3 \mathrm{ml}$ of 2 YT (1.6\% tryptone, $1 \%$ yeast extract and $0.5 \%$ $\mathrm{NaCl}$ with or without $1 \mathrm{mM}$ IPTG) containing the same antibiotics, the colonies were plated on $2 \mathrm{YT}$ 
agar plates containing ampicillin alone $(100 \mu \mathrm{g} / \mathrm{ml})$ and on plates containing both tetracycline $(25 \mu \mathrm{g} /$ $\mathrm{ml})$ and ampicillin $(100 \mu \mathrm{g} / \mathrm{ml})$. At the same time, from the last liquid steps, we isolated (parallel to inoculating the plates) plasmids to control form (size) actually presented inside the cells. An $A p^{R}$ and $\mathrm{Tc}^{\mathrm{S}}$ phenotype shows that the incoming plasmid harbors a gene that complements EcoSSB. On the other hand, maintenance of $\mathrm{Tc}^{\mathrm{R}}, \mathrm{Ap}^{\mathrm{R}}$ phenotype indicates that the tester plasmid is unable to complement EcoSSB.

Protein sequence analysis. The amino-acid sequences of DgrSSB and DprSSB were analysed using standard protein-protein BLAST and RPS-BLAST. Multiple sequence alignment was generated by using the program ClustalX. The results were prepared using the editor program Gendoc (copyright Karl Nicholas).

\section{RESULTS}

\section{Comparison of Thermus/Deinococcus SSBs}

The nucleotide sequence of the PCR products obtained by amplification of the region between $r p s F$ and $r p s R$ genes revealed a contiguous ORF that encodes a complete SSB protein of $D$. grandis or $D$. proteolyticus. The monomer of the DgrSSB protein contains 296 amino-acid residues and DprSSB 291 amino-acid residues and they are five and ten amino-acid residues respectively, shorter than the largest bacterial SSB polypeptide identified up to date $(D$. radiodurans SSB contains 301 amino-acid residues). Analysis of the primary structures revealed the presence of two putative OB-fold domains that is common to all known SSB proteins of the Deinococcus/Thermus phylum. Figure 1 shows an alignment of $D$. radiodurans, $D$. geothermalis, $D$. radiopugnans, D. grandis, D. proteolyticus, T. aquaticus and T. thermophilus SSBs. Of these, the Thermus SSBs have been first characterized extensively biochemically and biophysically as a prototype for SSB proteins in homodimeric form, where each SSB monomer contains two OB-folds (Dąbrowski et al., 2002a; $2002 b)$. Two years ago SSB from D. radiodurans was also characterized (Bernstein et al., 2004; Eggington et al., 2004). Analysis shows that Thermus/Deinococcus SSBs are highly conserved. Protein sequence of DgrSSB shares $82 \%$ identity and $89 \%$ similarity to D. radiodurans $\mathrm{R} 1 \mathrm{SSB}, 44 \%$ identity and $63 \%$ similarity to T. thermophilus HB-8 SSB, 44\% identity and $62 \%$ similarity to $T$. thermophilus VK-1 SSB and $45 \%$ identity and $63 \%$ similarity to T. aquaticus YT-1 SSB protein. DprSSB protein shares $69 \%$ identity and $82 \%$ similarity to D. radiodurans R1 SSB and $43 \%$ identity and $63 \%$ similarity to $T$. thermophilus HB-8 SSB, $43 \%$ identity and $62 \%$ similarity to T. thermophilus VK-1 SSB and 43\% identity and 61\% similarity to T. aquaticus YT-1 SSB (Fig. 1). For both examined proteins the $\mathrm{N}$-terminal fragment shares two, three times higher identity and similarity to adequate fragment of $D$. radiodurans $\mathrm{R} 1$ than to E. coli SSB protein. The C-terminal fragments of $D g r S S B$ and DprSSB proteins are essentially more identical to EcoSSB. On the other hand, the N-terminal fragments of DgrSSB and DprSSB proteins do not share such a high degree of homology with EcoSSB. In the formation of homodimers, Thermus/Deinococcus SSBs maintain the bacterial trend of four OB folds per SSB protein oligomer. The structure of $\mathrm{DgrSSB}$ and DprSSB proteins is consistent with and reinforces the close phylogenetic relationship between Deinococcus and bacteria of the Thermus group of extremophiles.

\section{Expression and purification of $\mathrm{DgrSSB}$ and $\mathrm{DprSSB}$}

Using the recombinant $\mathrm{pBADDgrSSB}$ or pBADDprSSB plasmid, excellent inducible expression of a protein of the predicted size was obtained (Fig. 2, lane 1 and 1A). Both proteins were expressed in a soluble form in the cytosol. The recombinant proteins were purified by a heat denaturation and two column chromatography steps as described in Materials and Methods. There is some reduction of contamination by the host proteins after the heat treatment step (Fig. 2, lane 2 and 2A). These results were not surprising as we described previously that isolated SSB protein from mesophilic $D$. radiopugnans bacteria was highly thermostable (Filipkowski et al., 2006b). The studied proteins also come from mesophilic microorganisms, for which the optimum temperature of growth is below $30^{\circ} \mathrm{C}(\mathrm{D}$. grandis). The applied overexpression system produces up to about $180 \mathrm{mg}$ purified DgrSSB or DprSSB protein from 1 liter of induced culture. These proteins after concentration were purified with about $99 \%$ purity (Fig. 2, lane 5 and $5 \mathrm{~A}$ ).

\section{Oligomerization status of DgrSSB and DprSSB}

Molecular masses of DgrSSB and DprSSB, as calculated from the amino-acid sequences, are 32.29 $\mathrm{kDa}$ and $31.33 \mathrm{kDa}$, respectively. To determine the oligomeric state of these proteins, they were analyzed by gel filtration chromatography. The DgrSSB and DprSSB proteins eluted from a calibrated gel filtration column with estimated sizes of about $53.07 \mathrm{kDa}$ and $61.06 \mathrm{kDa}$ (not shown), consistent with homodimeric subunit composition in solution. This confirmed our prediction that DgrSSB and DprSSB exist in solution as a homodimers. 

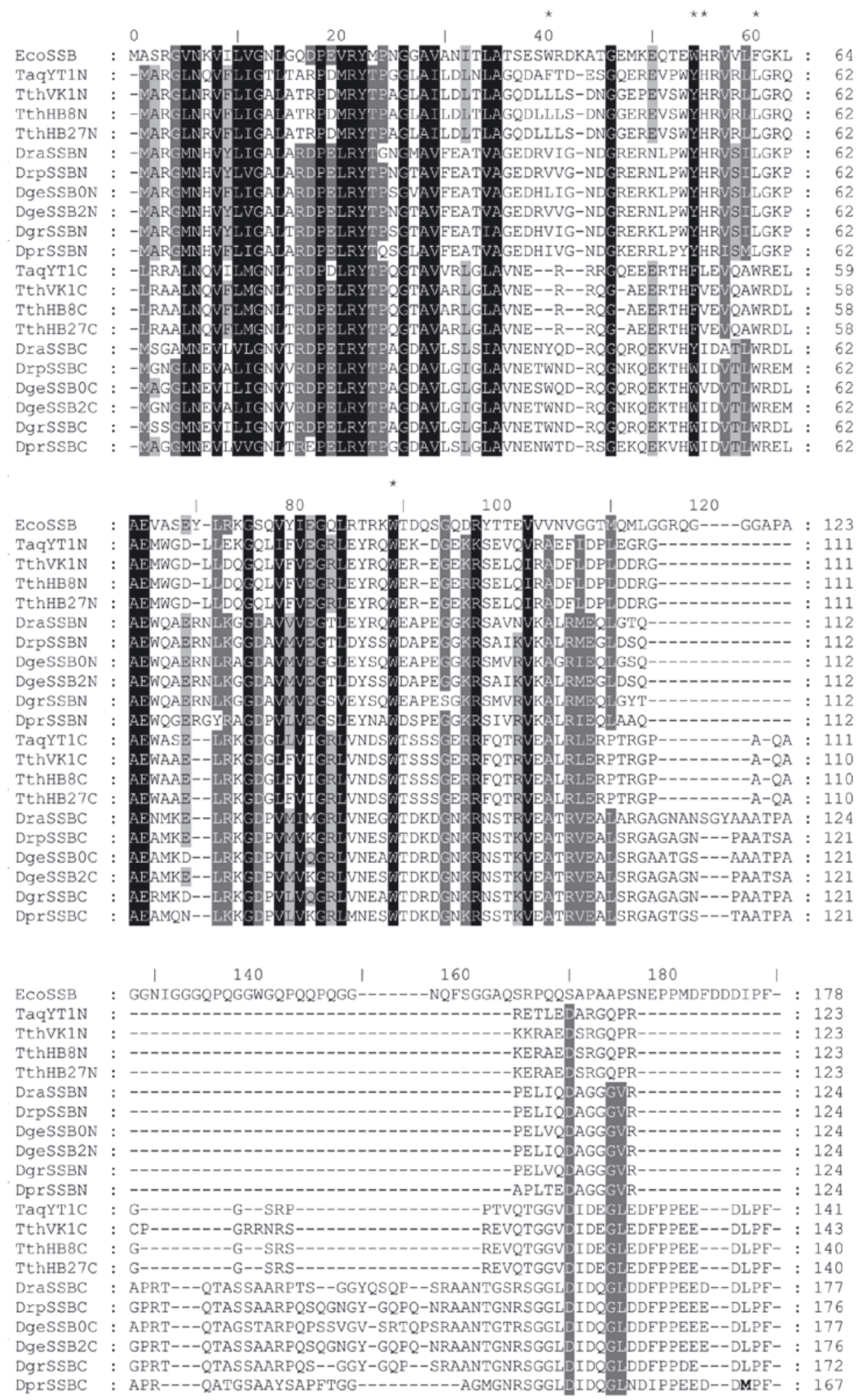

Figure 1. Multiple amino-acid sequence alignment of SSB-like proteins.

Alignment was performed by dividing amino-acids into six similarity groups: group 1, V, L, I and M; group 2, W, F and Y; group 3, E and D; group 4, K and R; group 5, Q and D; group 6, S and T. Description of similarity: white fonts on black boxes, $100 \%$ identity; white fonts on grey boxes, similarity $<80 \%$; black fonts on grey boxes, similarity $<60 \%$. Asterisks indicate conserved amino acids in the EcoSSB sequence that is engaged in stacking interaction with bases of ssDNA (W41, W55, F61 and W89) and stabilization of the tetramer (H56). Abbreviations: EcoSSB, E. coli K-12; TaqYT1, T. aquaticus strain YT-1; TthHB8, T. thermophilus strain HB-8; TthHB27, T. thermophilus strain HB-27; TthVK1, T. thermophilus strain VK-1; DraSSB, D. radiodurans strain R1; DrpSSB, D. radiopugnans; DgeSSB0, D. geothermalis strain 11300; DgeSSB2, D. geothermalis strain 11302; DgrSSB, D. grandis; DprSSB, D. proteolyticus; N, N-terminal ssDNA-binding domain; C, C-terminal ssDNA-binding domain. 


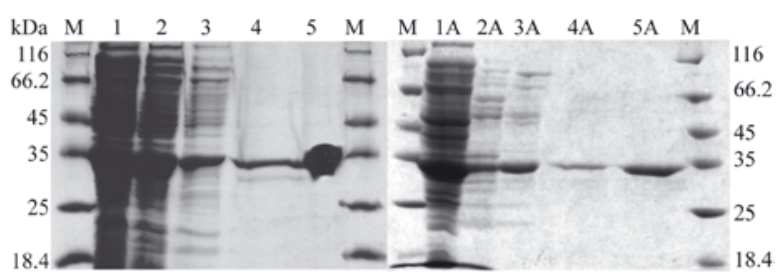

Figure 2. SDS-electrophoresis in $12 \%$ polyacrylamide gel of the fractions obtained by expression and purification of the recombinant DgrSSB from $E$. coli TOP10F'+pBAD-DgrSSB (left panel) and DprSSB from E. coli TOP10F'+pBAD-DprSSB ("A"-right panel).

Lane M, LMW markers (Fermentas), the molecular mass of proteins are marked; lane 1 and $1 \mathrm{~A}$, soluble protein cell extracts after arabinose induction of protein expression; lane 2, DgrSSB and 2A DprSSB, after heat treatment; lane 3 DgrSSB and 3A DprSSB after chromatography on QAE-cellulose column; lane 4 DgrSSB and 4A DprSSB after chromatography on ssDNA-cellulose column; lane 5 concentrated DgrSSB protein and 5A concentrated DprSSB protein.

DNA binding monitored by retardation assay

To determine the ability of DgrSSB and DprSSB proteins to bind to ssDNA, we carried out agarose gel mobility assays with fluorescein-labelled ss dT-oligonucleotides 35, 60, 70, 76 nucleotides in length (Fig. 3). For $(\mathrm{dT})_{35}$ and $(\mathrm{dT})_{60}$ there was a one complex regardless of protein concentration. A band of identical mobility was observed for $(\mathrm{dT})_{70}$ and $(\mathrm{dT})_{76}$ at low protein concentrations, but a second band with a lower mobility was observed at high protein concentrations. These results suggest that $D g r S S B$ and DprSSB proteins bind to $(\mathrm{dT})_{35}$ and $(\mathrm{dT})_{60}$ as a single protein molecule whereas two SSB molecules bind to $(\mathrm{dT})_{70}$ and $(\mathrm{dT})_{76}$. The implications are that the minimum length of DNA that is required for efficient binding is between 30 and 35 nucleotides long.

DNA binding monitored by quenching of intrinsic tryptophan fluorescence

To explore the binding properties of examined SSB proteins further, we made use of fluorescence spectroscopy (Fig. 4). All homotetrameric and homodimeric SSB proteins studied so far show a dramatic decrease of tryptophan fluorescence when binding to ssDNA. With an excitation wavelength of $295 \mathrm{~nm}$, the emission spectrum of SSB-like proteins at $25^{\circ} \mathrm{C}$ had a maximum at $348 \mathrm{~nm}$, consistent with tryptophan fluorescence. On addition of a saturating quantity of ssDNA, the intrinsic fluorescence at $348 \mathrm{~nm}$ was quenched in $76 \%$ by DgrSSB and in $85 \%$ by $\mathrm{DprSSB}$. The estimated size of ssDNA binding site in the presence of $2 \mathrm{mM}$ and $100 \mathrm{mM}$ of $\mathrm{NaCl}$ for DgrSSB and DprSSB proteins was about of $35 \pm 2 \mathrm{nt}$. And practically binding-mode transition has not been observed when changing the ionic environ-

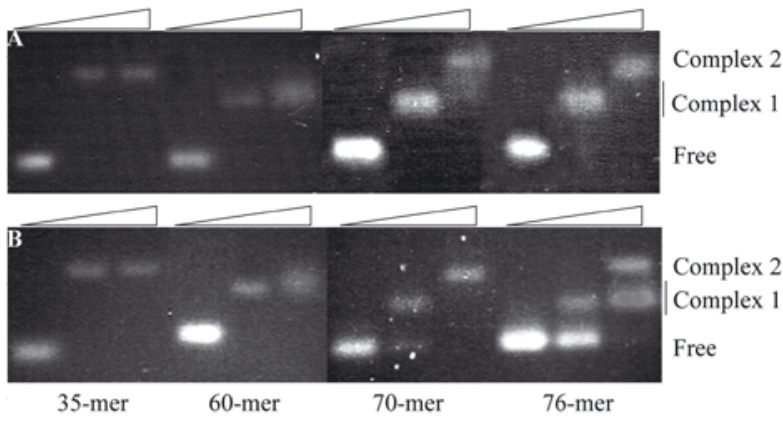

Figure 3. Binding of DgrSSB (A) and DprSSB (B) to single-stranded oligonucleotides - gel mobility shift assays.

ment from low $(2 \mathrm{mM} \mathrm{NaCl})$ to high salt $(100 \mathrm{mM}$ $\mathrm{NaCl})$.

\section{Thermostability}

The half-lifes of ssDNA-binding activity of DgrSSB, DprSSB, DraSSB and TaqSSB proteins at different temperatures and times were determined by gel mobility shift assay of poly $(\mathrm{dT})_{35}$ in $2 \%$ agarose gel (Fig. 5). The half-lives of ssDNA-binding activity of $\mathrm{DgrSSB}$ are $40 \mathrm{~min}$ at $55^{\circ} \mathrm{C}$ and $1 \mathrm{~min}$ at $65^{\circ} \mathrm{C}$. DprSSB is only slightly more thermostable than DgrSSB with $90 \mathrm{~min}$ at $55^{\circ} \mathrm{C}$ and $1 \mathrm{~min}$ at $67.5^{\circ} \mathrm{C}$. Previously described SSB proteins (TaqSSB, DgeSSB and DrpSSB) have higher thermostbility than SSB proteins examined in this work (Dąbrowski et al., 2002a; 2002b; Filipkowski et al., 2006a; 2006b).

\section{DgrSSB and DprSSB can replace EcoSSB in vivo}

In the complementation experiments, we attempted to replace the resident plasmid (pRPZ146, ori $\mathrm{ColEl}, \mathrm{Tc}^{\mathrm{R}}$, harboring a wild-type ssb gene) from E. coli RDP268(DE3) ( $\Delta s s b::$ Kan) with the plasmids harboring test ssb genes (pET23D $(+) D g r S S B$, pET23D(+)DprSSB or pET23D(+)EcoSSB, ori ColEl,

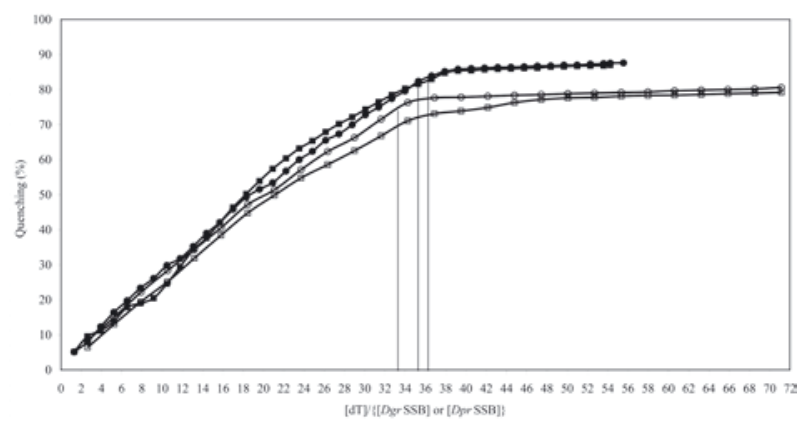

Figure 4. Inverse fluorescence titration of DgrSSB or DprSSB with $(\mathrm{dT})_{60}$.

A $1 \mathrm{nM}$ sample of SSB protein (homodimer) was titrated with $(\mathrm{dT})_{60}$ at $2 \mathrm{mM}$ (empty square) and $100 \mathrm{mM} \mathrm{NaCl}$ (empty circle) in standard buffer B and the same for DprSSB (filled figures). 


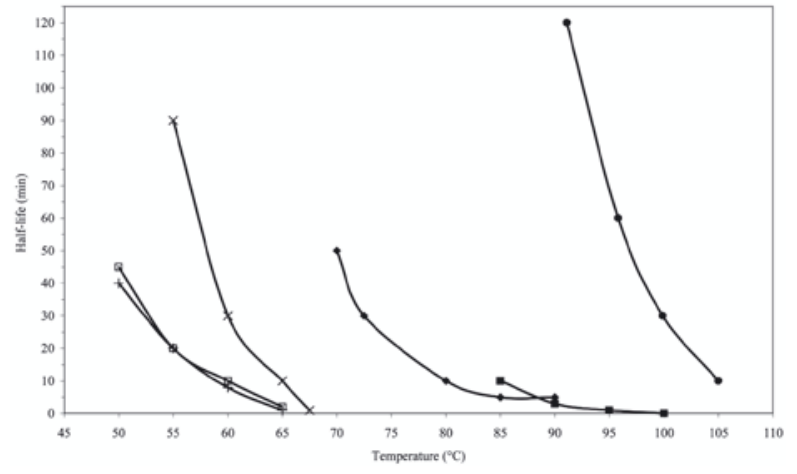

Figure 5. Half-lives of ssDNA-binding activity of DraSSB ( $\square), \operatorname{DgrSSB}(+), \operatorname{DprSSB}(\times), \operatorname{DgeSSB}(\diamond), \operatorname{TaqSSB}(\square)$ and DrpSSB $(\bullet)$.

$A p^{R}$ ). Since SSB is an essential protein, success in replacement of the original $\mathrm{Tc}^{\mathrm{R}}$ plasmid by the incoming $\mathrm{Ap}^{\mathrm{R}}$ plasmid, resulting in a $\mathrm{Tc}^{\mathrm{S}}, \mathrm{Ap}^{\mathrm{R}}$ phenotype, shows that the test SSB complements the $\Delta s s b$ strain of E. coli. The pET23D(+)EcoSSB was used as a control to assess the efficacy of the complementation assay. After transformation of modified E. coli cells with pET23D(+)DgrSSB, pET23D(+)DprSSB or pET23D $(+) E c o S S B$, which encodes resistance to ampicillin, and subsequent inoculations, we could isolate clones that showed resistance to ampicillin and kanamycin but not to tetracycline. These clones must have lost the pRPZ146 plasmid encoding EcoSSB. Additionally, an analysis of plasmid DNA was carried out after plasmid isolation from tested strains. The results confirmed the presence of appropriate plasmids. Interestingly, under the same assay conditions, we obtained better growth of cultures containing pET23D $(+) D g r S S B$ or pET23D $(+) D p r S S B$ than with pET23D $(+) E c o S S B$ and the results were independent of IPTG induction of SSB expression.

\section{DISCUSSION}

In this article, we describe the identification, purification and characterization of SSB-like proteins from the mesophilic D. grandis and D. proteolyticus microorganisms. From sequence analysis, it is obvious that two ssDNA-binding domains in one mono- mer of these proteins possess a canonical oligonucleotide binding fold, very similar to the homology observed in the structure of $D$. radiodurans SSB (Bernstein et al., 2004). Gel filtration chromatography experiments of DgrSSB and DprSSB clearly showed that these proteins form dimers in solution.

We showed that the binding of DgrSSB or DprSSB to ssDNA results in a quench of tryptophan fluorescence at over $76 \%$ under all salt conditions used. The binding site size of DgrSSB or DprSSB is of about $35 \mathrm{nt}$ per homodimer and is only slightly salt dependent. For EcoSSB, at least two distinctly different DNA-binding modes have been described (Lohman \& Overman 1985). Whereas under high salt conditions, $65 \mathrm{nt}$ bind per EcoSSB tetramer with almost $90 \%$ fluorescence quench, under low salt conditions $35 \mathrm{nt}$ are sufficient to saturate the protein and quench its fluorescence by only $53 \%$. The distinctly different binding mode of EcoSSB under high salt conditions could not be observed for DgrSSB and DprSSB, as for all known Deinococcus/Thermus SSBs. Based on the structure of a complex of EcoSSB and two molecules of $(\mathrm{dC})_{35}$, a model of the low salt binding mode of EcoSSB has been proposed (Raghunathan et al., 2000). In this model, the Trp-54 residues of only two subunits of EcoSSB are involved in DNA binding. The most important difference between the Deinococcus/Thermus SSBs and EcoSSB lies in the fact that Deinococcus/Thermus SSBs contain two instead of one DNA-binding domain per monomer. E. coli SSB base-stacking residues are Trp40, Trp-54, Phe-60, and Trp-88. The related residues in DgrSSB are Val-40, Tyr-53 (proceeds by Trp-52), Leu-59 and Trp-88 in the N-terminal OB domain. The same residues are present in DprSSB with only one exception: Tyr-53 is proceeds by Tyr-52. In the C-terminal OB domain there are Trp-164 (Tyr-164 in DraSSB), Trp-177 (Tyr-164 in DraSSB), Trp-183, and Trp-210 for both DgrSSB and DprSSB. Altogether, the C-terminal OB domain in the examined proteins contains homologous residues to the four aromatic base-stacking residues responsible for DNA binding by EcoSSB and only two of these residues are conserved in the N-terminal OB domain of the proteins (Fig. 1). A highly conserved His-55, important for homotetramerization of EcoSSB, is homologous to

Table 1. Percentages amino acid content of EcoSSB, DraSSB, DrpSSB, DgeSSB0, DgeSSB2, DgrSSB and DprSSB proteins.

\begin{tabular}{lcccccccccccccccccccc}
\hline $\begin{array}{l}\text { SSB } \\
\text { Protein }\end{array}$ & Ala & Arg & Asn & Asp & Cys & Gln & Glu & Gly & His & Ile & Leu & Lys & Met & Phe & Pro & Ser & Thr & Trp & Tyr & Val \\
\hline EcoSSB & 7.30 & 5.60 & 4.50 & 4.50 & 0.00 & 10.10 & 5.60 & 16.30 & 0.60 & 2.80 & 4.50 & 3.40 & 3.40 & 2.20 & 6.70 & 5.60 & 5.10 & 2.20 & 2.20 & 7.30 \\
DraSSB & 11.3 & 8.97 & 5.65 & 6.31 & 0.00 & 4.32 & 7.64 & 11.3 & 1.00 & 2.99 & 7.31 & 2.99 & 2.99 & 1.00 & 4.98 & 4.65 & 4.98 & 1.66 & 2.99 & 6.98 \\
DrpSSB & 10.33 & 8.00 & 6.33 & 6.67 & 0.00 & 3.67 & 7.67 & 12.33 & 1.00 & 2.33 & 7.33 & 4.33 & 2.67 & 1.00 & 5.33 & 4.33 & 5.00 & 2.33 & 2,00 & 7.33 \\
DgeSSB0 & 10.96 & 9.30 & 3.32 & 6.31 & 0.00 & 4.65 & 7.64 & 11.63 & 1.33 & 1.99 & 8.64 & 2.99 & 1.99 & 1.33 & 5.65 & 4.98 & 5.65 & 2.33 & 1.33 & 7.97 \\
DgeSSB2 & 10.33 & 8.00 & 6.33 & 6.67 & 0.00 & 3.67 & 7.67 & 12.33 & 1.00 & 2.33 & 7.33 & 4.33 & 2.67 & 1.00 & 5.33 & 4.33 & 5.00 & 2.33 & 2.00 & 7.33 \\
DgrSSB & 9.46 & 9.12 & 4.39 & 6.76 & 0.00 & 5.05 & 7.09 & 11.82 & 1.35 & 2.70 & 7.77 & 3.38 & 2.70 & 1.01 & 5.74 & 5.07 & 5.74 & 2.36 & 2.36 & 7.09 \\
DprSSB & 10.65 & 7.56 & 4.12 & 5.84 & 0.00 & 2.75 & 8.25 & 12.71 & 1.37 & 2.75 & 8.59 & 4.12 & 3.09 & 1.37 & 5.15 & 5.15 & 5.15 & 2.06 & 2.41 & 6.87 \\
\hline
\end{tabular}


the corresponding His residue present only in the Nterminal domains of $D g r S S B$ and DprSSB (as in other Thermus/Deinococcus SSBs). Two binding domains ( $\mathrm{N}$ - and C-terminal OB-folds) could have evolved separately and amino acids known to be involved in DNA binding were conserved more in the C-terminal OB-fold than in the N-terminal one (see Fig. 1).

DgrSSB and DprSSB, like other SSB proteins, contain in the C-terminal part a proline- and glycine-rich region, which is expected to be very flexible. Similarly to the other homologs, in DgrSSB and DprSSB the proline- and glycine-rich region is followed by the last 10 amino acids, which are highly conserved in this class. For EcoSSB, it could be shown that this region is responsible for the interaction with other proteins involved in DNA metabolism (Kelman et al., 1998; Genschel et al., 2000; Handa et al., 2001; Witte et al., 2003). It has been speculated that the glycine- and proline-rich region acts as a spacer between the DNA-binding domain and the negatively charged region of the last 10 amino acids (Curth et al., 1996). Thus, the formation of complexes between SSB proteins and their interaction partners could be facilitated by an easy access of this region.

EcoSSB is essential for the survival of the E. coli cell (Porter et al., 1990). Using an E. coli ssb mutant strain, we could show that DgrSSB and DprSSB can take over the function of EcoSSB in vivo. Since DgrSSB or DprSSB (like as other SSBs from Deinococcus/Thermus phylum) as dimers contain only two instead of four C-termini, two C-termini seem to suffice for the in vivo function of SSB. It has been speculated that the loss of two of the four C-termini in DraSSB may lead to functional differences (Eggington et al., 2004). Our data clearly show that such functional differences, even if they exist, do not play an important role in the function of SSB in vivo.

Our observations revealed that DgrSSB and DprSSB (like other known Deinococcus/Thermus SSBs) seem to be a normal representatives of bacterial SSB proteins. Except for the absence of salt binding-mode transition, Deinococcus/Thermus SSBs have all the essential properties of the prototype EcoSSB, including the ability to take over the vital role of EcoSSB in E. coli cells. The question as to why evolution chose to create homodimeric bacterial SSB proteins remains open.

\section{Acknowledgements}

The work was supported by the Gdańsk University of Technology. We thank Dr. M. Olszewski for TaqSSB protein used in this work and R. D. Porter for E. coli RDP268 strain used in complementation analysis experiments.

\section{REFERENCES}

Bernstein DA, Eggington JM, Killoran MP, Misic AM, Cox MM, Keck JL (2004) Crystal structure of the Deinococcus radiodurans single-stranded DNA-binding protein suggests a mechanism for coping with DNA damage. Proc Natl Acad Sci USA 101: 8575-8580.

Bochkarev A, Pfuetzner RA, Edwards AM, Frappier L (1997) Structure of the single-stranded-DNA-binding domain of replication protein A bound to DNA. Nature 385: 176-181.

Bochkarev A, Bochkareva E, Frappier L, Edwards AM (1999) The crystal structure of the complex of replication protein A subunits RPA32 and RPA14 reveals a mechanism for single-stranded DNA binding. EMBO J 18: 4498-4504.

Curth U, Genschel J, Urbanke C, Greipel J (1996) In vitro and in vivo function of the C-terminus of Escherichia coli single-stranded DNA binding protein. Nucleic Acids Res 24: 2706-2711.

Dąbrowski S, Kur J (1999) Cloning, overexpression, and purification of the recombinant His-tagged SSB protein of Escherichia coli and use in polymerase chain reaction amplification. Protein Expr Purif 16: 96-102.

Dąbrowski S, Olszewski M, Piątek R, BrillowskaDąbrowska A, Konopa G, Kur J (2002a) Identification and characterization of single-stranded-DNA-binding proteins from Thermus thermophilus and Thermus aquaticus - new arrangement of binding domains. Microbiology 148: 3307-3315.

Dąbrowski S, Olszewski M, Piątek R, Kur J (2002b) Novel thermostable ssDNA-binding proteins from Thermus thermophilus and T. aquaticus - expression and purification. Protein Expr Purif 26: 131-138.

de Groot A, Chapon V, Servant P, Christen R, Saux MF, Sommer S, Heulin T (2005) Deinococcus deserti sp. nov., a gamma-radiation-tolerant bacterium isolated from the Sahara Desert. Int J Syst Evol Microbiol 55: 2441-2446.

Eggington JM, Haruta N, Wood EA, Cox MM (2004) The single-stranded DNA-binding protein of Deinococcus radiodurans. BMC Microbiol 4: 2.

Ferreira AC, Nobre MF, Rainey FA, Silva MT, Wait R, Burghardt J, Chung AP, da Costa MS (1997) Deinococcus geothermalis sp. nov. and Deinococcus murrayi sp. nov., two extremely radiation-resistant and slightly thermophilic species from hot springs. Int J Syst Bacteriol 47: 939-947.

Filipkowski P, Duraj-Thatte A, Kur J (2006a) Novel thermostable single-stranded DNA-binding protein (SSB) from Deinococcus geothermalis. Arch Microbiol 186: 129-137.

Filipkowski P, Koziatek M, Kur J (2006b) A highly thermostable, homodimeric single-stranded DNA-binding protein from Deinococcus radiopugnans. Extremophiles. 10: 607-614.

Genschel J, Curth U, Urbanke C (2000) Interaction of E. coli single-stranded DNA binding protein (SSB) with exonuclease I. The carboxy-terminus of SSB is the recognition site for the nuclease. Biol Chem 381: 183-192.

Handa P, Acharya N, Varshney U (2001) Chimeras between single-stranded DNA-binding proteins from Escherichia coli and Mycobacterium tuberculosis reveal that their C-terminal domains interact with uracil DNA glycosylases. J Biol Chem 276: 16992-16997.

Hirsch P, Gallikowski CA, Siebert J, Peissl K, Kroppenstedt R, Schumann P, Stackebrandt E, Anderson R (2004) Deinococcus frigens sp. nov., Deinococcus saxicola sp. nov., and Deinococcus marmoris sp. nov., low tempera- 
ture and draught-tolerating, UV-resistant bacteria from continental Antarctica. Syst Appl Microbiol 27: 636-645.

Jędrzejczak R, Dauter M, Dauter Z, Olszewski M, Długołęcka A, Kur J (2006) Structure of the singlestranded DNA-binding protein SSB from Thermus aquaticus. Acta Crystallogr D Biol Crystallogr 62: 14071412.

Kelman Z, Yuzhakov A, Andjelkovic J, O’Donnell M (1998) Devoted to the lagging strand - the $\chi$-subunit of DNA polymerase III holoenzyme contacts SSB to promote processive elongation and sliding clamp assembly. EMBO J 17: 2436-2449.

Kur J, Olszewski M, Długołęcka A, Filipkowski P (2005) Single-stranded DNA-binding proteins (SSBs) - sources and applications in molecular biology. Acta Biochim Polon 52: 569-574.

Lohman TM, Overman LB (1985) Two binding modes in Escherichia coli single strand binding protein-single stranded DNA complexes. Modulation by $\mathrm{NaCl}$ concentration. J Biol Chem 260: 3594-3603.

Murzin AG (1993) OB(oligonucleotide/oligosaccharide binding)-fold: common structural and functional solution for non-homologous sequences. EMBO J 12: 861867.

Omelchenko MV, Wolf YI, Gaidamakova EK, Matrosova VY, Vasilenko A, Zhai M, Daly MJ, Koonin EV, Makarova KS (2005) Comparative genomics of Thermus thermophilus and Deinococcus radiodurans: divergent routes of adaptation to thermophily and radiation resistance. BMC Evol Biol 5: 57.

Porter RD, Black S (1991) The single-stranded-DNA-binding protein encoded by the Escherichia coli $\mathrm{F}$ factor can complement a deletion of the chromosomal ssb gene. J Bacteriol 173: 2720-2723.

Porter RD, Black S, Pannuri S, Carlson A (1990) Use of the Escherichia coli SSB gene to prevent bioreactor takeover by plasmidless cells. Biotechnology (N Y) 8: 47-51.
Raghunathan S, Kozlov AG, Lohman TM, Waksman G (2000) Structure of the DNA binding domain of E. coli SSB bound to ssDNA. Nat Struct Biol 7: 648-652.

Shamoo Y, Friedman AM, Parsons MR, Konigsberg WH, Steitz TA (1995) Crystal structure of a replication fork single-stranded DNA binding protein (T4 gp32) complexed to DNA. Nature 376: 362-366.

Stassen MJ, Bailey D, Nelson S, Chinwalla V, Harte PJ (1995) The Drosophila trithorax proteins contain a novel variant of the nuclear receptor type DNA binding domain and an ancient conserved motif found in other chromosomal proteins. Mech Dev 52: 209-223.

Wadsworth RI, White MF (2001) Identification and properties of the crenarchaeal single-stranded DNA binding protein from Sulfolobus solfataricus. Nucleic Acids Res 29: 914-920.

Webster G, Genschel J, Curth U, Urbanke C, Kang C, Hilgenfeld R (1997) A common core for binding single-stranded DNA: structural comparison of the singlestranded DNA-binding proteins (SSB) from E. coli and human mitochondria. FEBS Lett 411: 313-316.

Williams KR, Konigsberg W (1978) Structural changes in the T4 gene 32 protein induced by DNA polynucleotides. J Biol Chem 253: 2463-2470.

Witte G, Urbanke C, Curth U (2003) DNA polymerase III chi subunit ties single-stranded DNA binding protein to the bacterial replication machinery. Nucleic Acids Res 31: 4434-4440.

Witte G, Urbanke C, Curth U (2005) Single-stranded DNAbinding protein of Deinococcus radiodurans: a biophysical characterization. Nucleic Acids Res 33: 1662-7160.

Yang C, Curth U, Urbanke C, Kang C (1997) Crystal structure of human mitochondrial single-stranded DNA binding protein at $2.4 \mathrm{~A}$ resolution. Nat Struct Biol 4: 153-157. 